\title{
Joint Optimization of FIR Prefilter and Channel Estimate for Sequence Estimation
}

\author{
Jan C. Olivier and Chengshan Xiao
}

\begin{abstract}
We provide simple analytical results for the coefficients of a finite-impulse response (FIR) prefilter and the effective channel impulse response (IR) for use in cellular communication systems. We show that using a FIR filter with both causal and anticausal filter taps, it is possible to find the jointly optimized impulse response, such that the signal-to-noise ratio is maximized in the least-squares sense. We show via computer simulation for 8-ary phase-shift keying in enhanced data rates for global evolution (EDGE) that the joint optimization of the prefilter and IR produces results similar to the minimum mean-square error decision-feedback equalizer prefilter in thermal noise, but yields gain in colored noise.
\end{abstract}

Index Terms-Channel estimation, maximum-likelihood detection, prefilter design, sequence estimation.

\section{INTRODUCTION}

$\mathbf{F}$ OR THIRD-GENERATION cellular communications systems based on time-division multiple-access (TDMA), the existing standards are being evolved to higher spectral efficiency [1]. Specifically, the modulation scheme has been changed to 8-ary phase-shift keying (8PSK), thus due to receiver complexity considerations, optimal maximum-likelihood sequence estimator (MLSE) equalization cannot be used as a symbol detector, as was the case for binary Gaussian minimum-shift keying (GMSK) modulation in the global system for mobile communications (GSM).

Suboptimal sequence estimation methods employing decision feedback are used instead, and the design of a prefilter able to yield a channel impulse response estimate with dominant leading taps is important [2]-[6]. In [6], it was shown that for the finite-impulse response (FIR) filter design under the minimum mean-square error (MMSE) criterion Cholesky factorization has to be used, the finite-dimensional analog of spectral factorization used in the infinite length case. The MMSE metric is not the only metric available for filter synthesis, as other authors, notably Salazar [7], Gerstacker [8], and Falconer et al. [9] have shown that the maximization of the signal-to-noise ratio (SNR) metric is also suitable. In this letter, we use the concept of maximization of SNR in the least-squares (LS) sense, combined with a FIR prefilter with both causal and anticausal taps. We so derive

Paper approved by Z. Kostic, the Editor for Wireless Systems of the IEEE Communications Society. Manuscript received January 30, 2001; revised July 18, 2001 and January 12, 2002.

J. C. Olivier is with the Nokia Research Center, Irving, TX 75039 USA (e-mail: jan.olivier@nokia.com).

C. Xiao is with the Department of Electrical and Computer Engineering, University of Missouri-Columbia, Columbia, MO 65211 USA (e-mail: xiaoc@missouri.edu).

Publisher Item Identifier 10.1109/TCOMM.2002.802539.

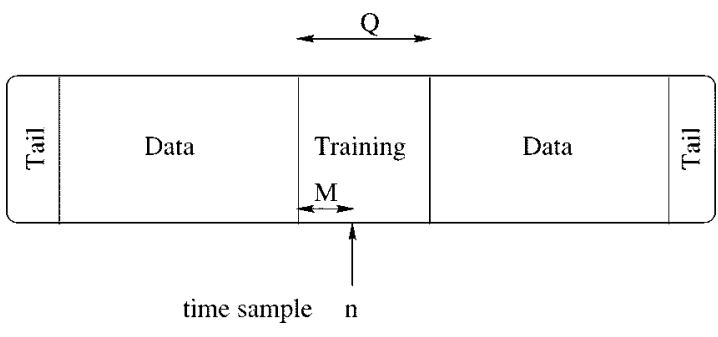

Fig. 1. A typical data burst as received in the receiver.

simple analytical expressions for the FIR filter coefficients, as well as a jointly optimized effective channel impulse response (IR) estimate. The analytical expressions for the IR estimate and FIR filter coefficients require only the inversion of two matrices, with sizes equal to the number of filter and IR taps chosen. Using our approach, apart from having to choose the length of the prefilter, there is no need for unknown delay parameters, nor do we require an estimation of the noise variance, or the channel estimate before the prefilter, as we do not require any expectation values to be estimated [8].

The letter is organized as follows. In Section II, we present the receiver and the joint optimizing scheme. Section III presents computer-simulated results achieved by this method and the MMSE method [5] for use in enhanced data rates for global evolution (EDGE) with 8PSK modulation. The conclusions are presented in Section IV.

\section{JoINT OPTIMIZATION OF FIR PREFILTER AND EFFECTIVE CHANNEL IR}

We make the assumption that the IR does not change significantly over a short burst of data, referred to as a data packet (Fig. 1). For a causal IR, let us define a training matrix, denoted by $\mathbf{S}$ and given in terms of the training symbols as shown in (2) at the bottom of the next page.

Next, we introduce the mathematical model of the receiver in baseband. Denote with $p[n]$ the prefiltered received signal in baseband (see Fig. 2) at a sampling rate high enough to provide for sufficient statistics for detection. Hence, we have in baseband

$$
p[n]=\sum_{l=0}^{M} h[l] d[n-l]+n_{s}[n]
$$

where in vector notation ${ }^{1} \mathbf{p}, \mathbf{h}, \mathbf{d}, \mathbf{n}_{\mathbf{s}}$ represent the prefiltered received signal, the effective channel IR after the prefilter $\mathbf{w}$, the transmitted data symbols to be estimated, and the prefiltered additive noise, respectively. $M+1$ is the length of the channel.

\footnotetext{
${ }^{1}$ Bold lower case symbols indicate column vectors, bold upper case symbols
} denote matrices. 


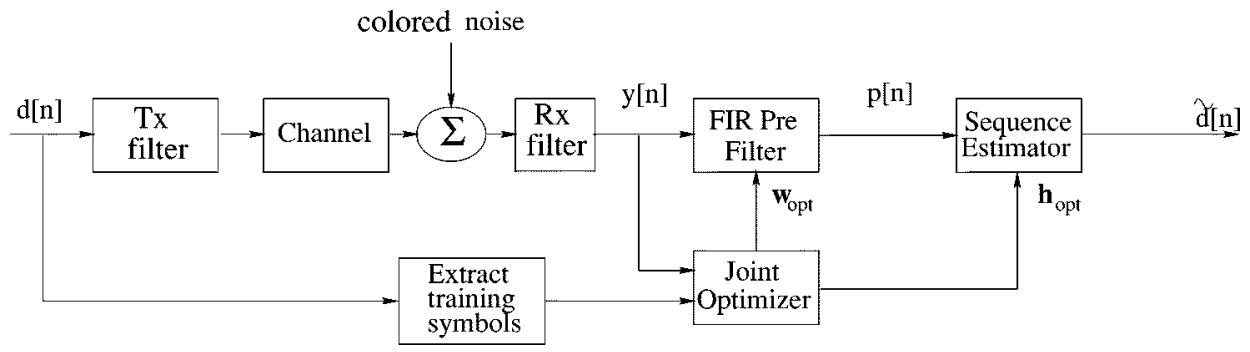

Fig. 2. Receiver with prefilter and the new joint optimizer.

Vector $\mathbf{d}$ contains $Q$ training symbols available inside the received data packet, as shown in Fig. 1. We also define a dominant IR tap matrix $\mathbf{Z}$, for $K+1$ dominant taps $(K<M)$ given by (3), shown at the bottom of the page. Let us also define a received matrix denoted $\mathbf{Y}$, formed with the received samples $\mathbf{y}$ and given by (4) at the bottom of the page.

Here $R+1$ is the number of taps we wish to use in the FIR prefilter, and $m$ is the number of taps that are anticausal. It is important to realize that we use only known training symbols and received symbols. Based on these matrices, we now present the expression for the SNR to be maximized as a ratio of two terms in the LS sense, one being the signal energy due to the dominant taps, and the other the noise energy.

$$
\max (\mathrm{SNR})=\max _{\mathbf{w}, \mathbf{h}}\left(\frac{\left\|\mathbf{h}^{T} \mathbf{Z}\right\|^{2}}{\left\|\mathbf{w}^{T} \mathbf{Y}-\mathbf{h}^{T} \mathbf{S}\right\|^{2}}\right) .
$$

It is clear that our definition of SNR to be maximized in the LS sense (5) is simple, in the sense that it does not require the estimation of the IR before the prefilter, nor does it require an unknown delay parameter to be optimized in addition to the SNR. The only additional parameter to be chosen is the length of the prefilter.

\section{A. Joint Optimization Procedure}

We now focus on finding analytical expressions for vectors $\mathbf{w}$ and $\mathbf{h}$ maximizing the SNR in (5) in the LS sense. We apply the separation of variables technique, starting by forcing the denominator in (5) to a minimum. ${ }^{2}$ Thus, we have

$$
\min \left\|\mathbf{w}^{\mathbf{T}} \mathbf{Y}-\mathbf{h}^{T} \mathbf{S}\right\|^{2} \Longrightarrow \mathbf{w}^{T}=\mathbf{h}^{T} \mathbf{S} \mathbf{Y}^{\dagger}\left(\mathbf{Y} \mathbf{Y}^{\dagger}\right)^{-1} .
$$

${ }^{2}$ In this paper we denote a complex conjugate with *, Hermitian with ${ }^{\dagger}$, and transpose with ${ }^{T}$.

$$
\mathbf{S}=\left(\begin{array}{cccccccc}
d[n] & d[n+1] & d[n+2] & d[n+3] & d[n+4] & d[n+5] & \cdots & d[Q-m] \\
d[n-1] & d[n] & d[n+1] & d[n+2] & d[n+3] & d[n+4] & \cdots & d[Q-1-m] \\
\vdots & \vdots & \vdots & \vdots & \vdots & \vdots & \vdots & \vdots \\
d[n-M] & d[n-M+1] & \cdots & \cdots & \cdots & \cdots & \cdots & d[Q-M-m]
\end{array}\right)
$$

$$
\mathbf{Z}=\left(\begin{array}{cccccccc}
d[n] & d[n+1] & d[n+2] & d[n+3] & d[n+4] & d[n+5] & \cdots & d[Q-m] \\
d[n-1] & d[n] & d[n+1] & d[n+2] & d[n+3] & d[n+4] & \cdots & d[Q-1-m] \\
\vdots & \vdots & \vdots & \vdots & \vdots & \vdots & \vdots & \vdots \\
d[n-K] & d[n-K+1] & \cdots & \cdots & \cdots & \cdots & \cdots & d[Q-K-m] \\
0 & 0 & \cdots & \cdots & \cdots & \cdots & \cdots & 0
\end{array}\right)
$$

$$
\mathbf{Y}=\left(\begin{array}{ccccccc}
y[n+m] & y[n+1+m] & y[n+2+m] & y[n+3+m] & y[n+4+m] & \cdots & y[Q] \\
y[n+m-1] & y[n+m] & y[n+m+1] & y[n+m+2] & y[n+m+3] & \cdots & y[Q-1] \\
y[n+m-2] & y[n+m-1] & \cdots & \cdots & \cdots & \cdots & y[Q-2] \\
\vdots & \vdots & \vdots & \vdots & \vdots & \vdots & \vdots \\
y[n+m-R] & y[n+m-R+1] & \cdots & \cdots & \cdots & \cdots & y[Q-R]
\end{array}\right)
$$


Upon substitution of (6) into (5) we can write

$$
\mathbf{h}_{\mathrm{opt}}=\arg \max _{\mathbf{h}}\left(\frac{\left\|\mathbf{h}^{T} \mathbf{Z}\right\|^{2}}{\left\|\mathbf{h}^{T} \mathbf{S}\left(\mathbf{Y}^{\dagger}\left(\mathbf{Y} \mathbf{Y}^{\dagger}\right)^{-1} \mathbf{Y}-\mathbf{I}\right)\right\|^{2}}\right)
$$

hence

$$
\mathbf{h}_{\text {opt }}=\arg \max _{\mathbf{h}}\left(\frac{\left\|\mathbf{h}^{T} \mathbf{Z}\right\|^{2}}{\left\|\mathbf{h}^{T} \mathbf{S P}\right\|^{2}}\right)
$$

where

$$
\mathbf{P}=\mathbf{I}-\mathbf{Y}^{\dagger}\left(\mathbf{Y} \mathbf{Y}^{\dagger}\right)^{-1} \mathbf{Y}
$$

$\mathbf{h}_{\mathrm{opt}}$ denotes the optimal effective channel IR. Now we may rewrite (8) as

$$
\mathbf{h}_{\mathrm{opt}}=\arg \max _{\mathbf{h}}\left(\frac{\mathbf{h}^{\dagger} \mathbf{Z}^{*} \mathbf{Z}^{\mathbf{T}} \mathbf{h}}{\mathbf{h}^{\dagger} \mathbf{S}^{*} \mathbf{P}^{*} \mathbf{P}^{\mathbf{T}} \mathbf{S}^{T} \mathbf{h}}\right) .
$$

Equation (10) is a generalized eigenvalue problem, and $\mathbf{h}_{\mathrm{opt}}$ is given by the eigenvector

$$
\mathbf{h}_{\mathrm{opt}}=\operatorname{eig}_{\lambda_{\max }}\left(\left[\mathbf{S}^{*} \mathbf{P}^{*} \mathbf{P}^{\mathbf{T}} \mathbf{S}^{\mathbf{T}}\right]^{-1} \mathbf{Z}^{*} \mathbf{Z}^{\mathbf{T}}\right)
$$

corresponding to the largest eigenvalue $\lambda_{\max }$. Thus, invoking (6), we can write the optimal FIR prefilter coefficients as

$$
\mathbf{w}_{\mathrm{opt}}^{T}=\mathbf{h}_{\mathrm{opt}}^{T} \mathbf{S} \mathbf{Y}^{\dagger}\left(\mathbf{Y} \mathbf{Y}^{\dagger}\right)^{-1} \cdot
$$

With the FIR filter and optimal IR coefficients now known, we can perform detection [10]. Note that this procedure will always whiten the noise after the prefilter, regardless of whether the noise was colored due to the receiver filter or was colored before the receiver filter, or both.

\section{Simulation Results}

In order to evaluate the performance of the results derived above and compare it to other techniques from the literature, we use EDGE and 8PSK modulation [1]. We select the typical urban (TU) model as a dispersive channel. We perform computer simulations for the decision-feedback equalizer (DFE) detector, using a five-tap IR with $M=4$, which is sufficient for TU. We compare our method to the MMSE-DFE prefilter [5]. We select the FIR prefilter with $R=6, m=6$, and $K=0$. These choices imply that we need to invert a $(7,7)$ matrix, and there are no additional noise covariance or channel impulse response estimations that need to be performed, as is the case for the MMSE-DFE prefilter.

In EDGE we have 26 training symbols, of which 22 are usable, as the TU model causes intersymbol interference (ISI) of four symbols before the prefilter. In the receiver, we use a square-root raised cosine (SRC) receiver filter with normalized bandwidth 0.67 and rolloff factor of 0.5 . The transmitter has a Gaussian filter as described by the GSM/EDGE standard. We simulate the case of AWGN and highly colored adjacent interference.

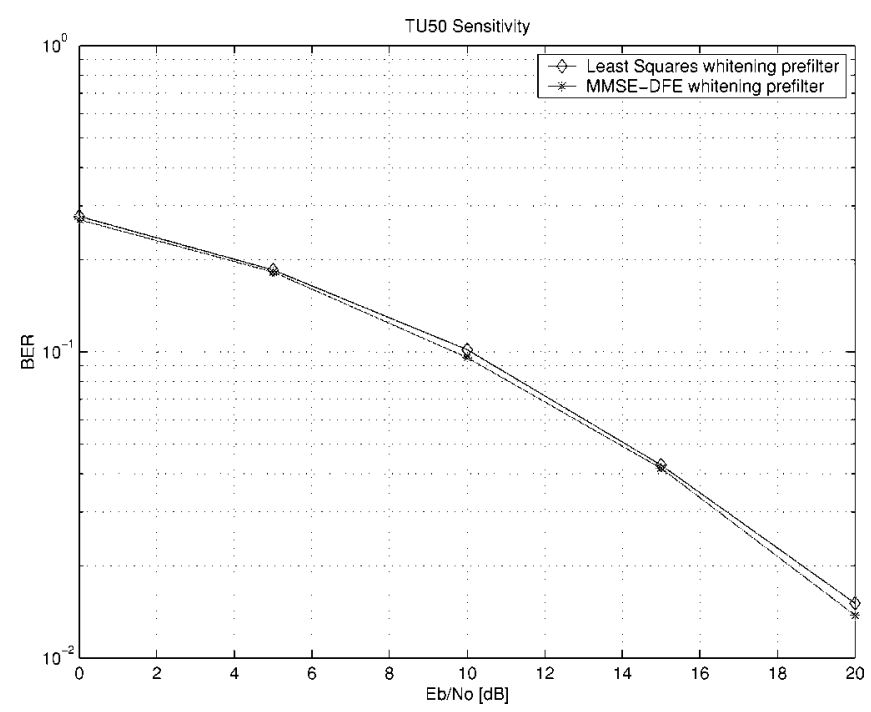

Fig. 3. BER results using a DFE detector for both the LS maximum SNR and MMSE-DFE prefilters in thermal noise.

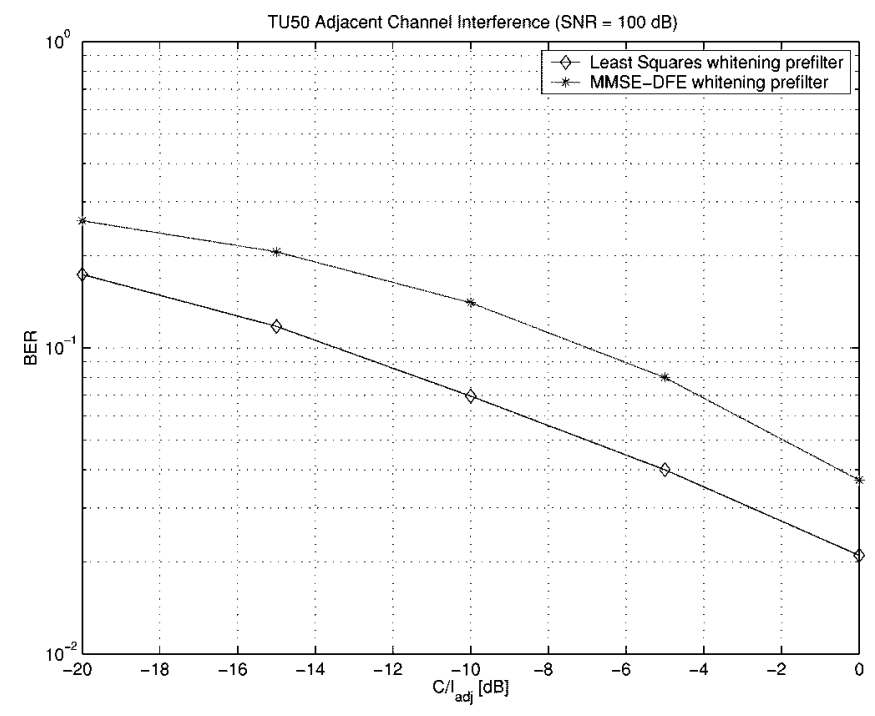

Fig. 4. BER results using a DFE detector for both the LS maximum SNR and MMSE-DFE prefilters in colored noise.

The bit error rate (BER) results obtained for the DFE detector are shown in Figs. 3 and 4. Clearly, overall BER results are similar for thermal noise, but the Max SNR LS prefilter yields gain in colored noise ${ }^{3}$.

\section{CONCLUSIONS}

We derived simple analytical expressions for the FIR prefilter and channel IR jointly optimized to maximize the SNR in the LS sense. We showed via computer simulations that our prefilter performs similarly compared to the MMSE-DFE prefilter in thermal noise, but yields gain in colored noise.

We showed that our approach, based on an LS metric, leads to a number of simplifications in the derived analytical expressions. For one, apart from having to choose the length of the prefilter, we do not need additional delay parameters, nor do we

${ }^{3}$ For the MMSE-DFE method the noise covariance matrix is estimated to enable noise whitening. 
need to estimate any expectation values or noise covariance matrices. The jointly optimized FIR prefilter and channel IR yields the best possible SNR we can achieve, given a small number of training symbols.

\section{ACKNOWLEDGMENT}

One of the authors, J. C. Olivier, wishes to thank C. Zhang at the Nokia Research Center for the many insightful discussions on the max SINR optimization problem.

\section{REFERENCES}

[1] R. Pirhonen, T. Rautava, and J. Penttinen, "TDMA convergence for packet data services," IEEE Pers. Commun. Mag., vol. 6, pp. 68-73, June 1999.

[2] W. H. Gerstacker and R. Schober, "Equalization for EDGE mobile communications," Electron. Lett., vol. 36, no. 2, pp. 189-191, Jan. 2000.
[3] M. Y. Eyuboglu and U. H. Qureshi, "Reduced state sequence estimation with set partitioning and decision feedback," IEEE Trans. Commun., vol. 36, pp. 13-20, Jan. 1988.

[4] A. Duel-Hallen and C. Heegard, "Delayed decision-feedback sequence estimation," IEEE Trans. Commun., vol. 37, pp. 428-435, May 1989.

[5] N. Al-Dhahir and J. M. Cioffi, "MMSE decision-feedback equalizers: Finite length results," IEEE Trans. Inform. Theory, vol. 41, pp. 961-975, July 1995.

[6] - "Fast computation of channel-estimate based equalizers in packet data transmission," IEEE Trans. Signal Processing, vol. 43, pp. 2462-2473, Nov. 1995.

[7] A. Salazar, "Design of transmitter and receiver filters for decision feedback equalization," Bell Syst. Tech. J., vol. 53, no. 3, Mar. 1974.

[8] W. H. Gerstacker and J. B. Huber, "Maximum SNR decision-feedback equalization with FIR filters: Filter optimization and a signal processing application," in Proc. IEEE Int. Conf. Communications, vol. 2, 1996, pp. $1188-1192$.

[9] D. Falconer and F. Magee, "Adaptive channel memory truncation for maximum-likelihood sequence estimation," Bell Syst. Tech. J., vol. 52, no. 9, pp. 1541-1562, Nov. 1973.

[10] T. Kailath and H. V. Poor, "Detection of stochatic processes," IEEE Trans. Inform. Theory, vol. 44, pp. 2230-2259, Oct. 1998. 